\title{
A loxoscelism case received therapeutic apheresis and hyperbaric oxygen therapy
}

\author{
Ali Cetinkaya, MD, Kaniye Aydin, MD, Hatice A. Sirakaya, MD, Rumeysa Yilmaz, MD.
}

\begin{abstract}

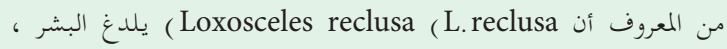

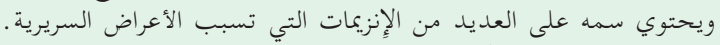

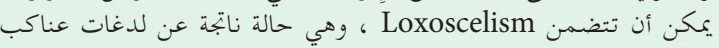

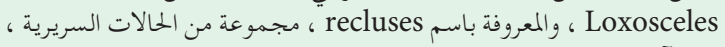

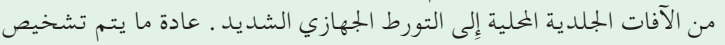

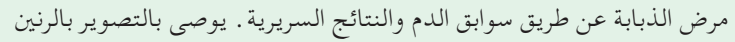

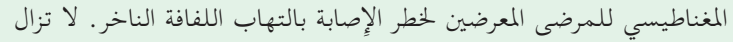

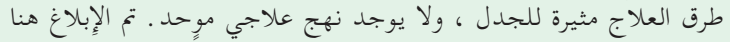

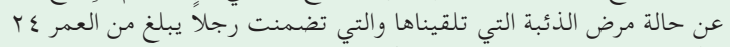

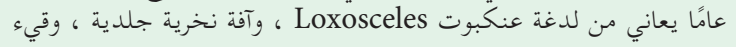

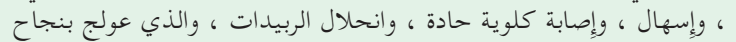

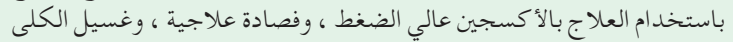

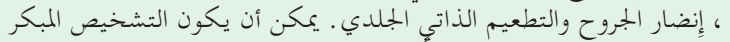

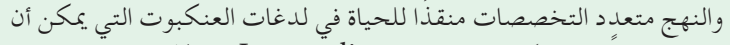

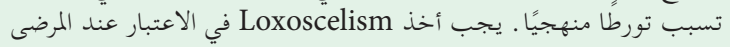

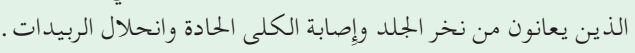

Loxosceles reclusa (L.reclusa) is known to bite humans, and its venom includes several enzymes that cause clinical symptoms. Loxoscelism, a condition due to being bitten by Loxosceles spiders, commonly known as recluses, can involve a range of clinical conditions, from local cutaneous lesions to severe systemic involvement. The diagnosis of loxoscelism is usually made by anamnesis and clinical findings. Magnetic resonance imaging is recommended for patients at high risk of necrotizing fasciitis. Treatment modalities are still controversial and there is no standardized treatment approach. Reported here, our case of loxoscelism involved a 24-year-old man presenting with a Loxosceles spider bite, dermonecrotic lesion, vomiting, diarrhea, acute renal injury, and rhabdomyolysis, who was successfully treated with hyperbaric oxygen therapy, therapeutic apheresis, hemodialysis, wound debridement, and cutaneous autografting. Early diagnosis and multidisciplinary approach can be life-saving in spider bites that can cause systemic involvement. Loxoscelism should be considered in patients with skin necrosis, acute renal injury, and rhabdomyolysis.
Keywords: loxosceles reclusa, therapeutic apheresis, hyperbaric oxygen therapy

$$
\text { Saudi Med J 2020; Vol. } 41 \text { (12): 1364-1368 }
$$
doi: 10.15537/smj.2020.12.25544

From the Department of Internal Medicine (Cetinkaya, Sirakaya, Yilmaz), and from the Department of Medical Intensive Care Unit (Aydin), Kayseri City Training and Research Hospital, Kayseri, Turkey.

Received 8th August 2020. Accepted 2nd November 2020.

Address correspondence and reprint request to: Dr. Kaniye Aydin, Department of Medical Intensive Care Unit, Kayseri City Training and Research Hospital, Kayseri, Turkey. E-mail: drkaniyeaydin@hotmail.com

ORCID ID: https://orcid.org/0000-0001-5538-3692

W ith thousands of species worldwide, spiders possess fangs containing venom glands, although only a few-for example, Loxosceles (examples, brown recluse), Latrodectus (example, black widow), and tarantulas-have fangs long enough to penetrate human skin. A particular possible result of being bitten by Loxosceles spiders is loxoscelism, a syndrome that in some cases can cause necrotizing skin lesions and systemic complications. Although itching, burning sensation, erythema, and local swelling are early symptoms of loxoscelism, the diagnosis is often overlooked. Moreover, because patients generally present to a hospital 24 to 48 hours after being bitten by spiders, delays in their treatment are frequent. Other complications can occur via venom-borne enzymes such as collagenase, peptidase, hyaluronidase, and sphingomyelinase D. In this report, we present findings, management and outcome of a patient with loxoscelism. This case report highlights that loxoscelism should be considered in the diagnosis in patients with skin necrosis due to spider bite, if there are also systemic complications, and that early diagnosis and multisystemic approach are life-saving. 
Case Report. Patient information. An otherwise healthy 24-year-old man presented to the emergency department with watery stool (such as, 15-20 defecations daily), vomiting, and abdominal pain. Once acute renal injury was detected in the patient, he was admitted to the medical intensive care unit (ICU). He was working as a server in an outdoor area of a café. He was suffering from a spider bite on his left arm for 3 days, and started complaining the following day. No abnormal finding was found in medical and family histories.

Clinical findings. In physical examination, vital signs were as follows: blood pressure, 65/44 $\mathrm{mmHg}$; heart rate, 125 beat per minute (no rhythm disorder); respiration rate, $30 / \mathrm{min}$; body temperature, $36.8^{\circ} \mathrm{C}$; arterial oxygen saturation, $90 \%$. Although a lesion 10 $\times 20 \mathrm{~cm}$ with central necrosis and peripheral erythema and edema was observable from the medial aspect in the middle third of the left upper arm (Figure 1), no other abnormal signs manifested in the systemic evaluation.

Diagnostic assessment. Table 1 summarizes the laboratory findings of the patient, whose Acute Physiology and Chronic Health Evaluation (APACHE) II score was 24 and Sequential Organ Failure Assessment (SOFA) score was 12 . He had no urine output. With current findings, he had diagnoses of acute kidney injury, rhabdomyolysis, and sepsis. Blood, urine, and wound swab cultures were taken. The result of the direct coombs test was positive, whereas that of the indirect coombs test was negative. No atypical cells and no evidence of hemolysis were observed in the peripheral blood smear. The complement 3 level was determined to be $0.75 \mathrm{~g} / \mathrm{L}$ (reference range: 0.9-1.8), and tests with hepatitis B surface antigen, anti-hepatitis $\mathrm{C}$, and anti-human immunodeficiency viruses also returned negative results. Values for amylase and lipase were within the normal reference range, and abdominal sonography showed a normal status. Sonography of the superficial tissue of the left upper arm; however, revealed edema in the skin and subcutaneous adipose tissue. He first described the spider to us. We looked at the pictures of the spiders that can be found in Turkey on the internet with him. He showed us the spider called L. reclusa and said that this spider species bit his left arm. Magnetic resonance imaging (MRI) of the left humerus showed edema in the soft tissue planes on the skin and subcutaneous region. His Laboratory Risk

Disclosure. Authors have no conflict of interests, and the work was not supported or funded by any drug company.
Indicator for Necrotizing Fasciitis (LRINEC) score, a scoring system designed to predict necrotizing fasciitis at admission, was 8 (it is based on white blood cell count, hemoglobin, serum sodium, creatinine, blood glucose, and C-reactive protein levels).

Therapeutic intervention. Antibiotic therapy was initiated, followed by a tetanus and diphtheria (TD) vaccine and fluid resuscitation. Because the

Table 1 - The patient's laboratory values on intensive care unit admission.

\begin{tabular}{|c|c|c|}
\hline Variables & $\begin{array}{l}\text { Reference range, } \\
\text { adults }\end{array}$ & $\begin{array}{c}\text { On } \\
\text { admission }\end{array}$ \\
\hline White blood cell $\left(10^{3} / \mu \mathrm{L}\right)$ & $4.5-10$ & 11.2 \\
\hline Hemoglobin (g/dL) & $13-17$ & 15.6 \\
\hline Platelet $\left(10^{3} / \mu \mathrm{L}\right)$ & $150-450$ & 103 \\
\hline Blood Urea Nitrogen (mg/dL) & $6-20$ & 88.2 \\
\hline Creatinine (mg/dL) & $0.7-1.2$ & 9.62 \\
\hline Sodium $(\mathrm{mmol} / \mathrm{L})$ & $136-145$ & 127 \\
\hline Potassium (mmol/L) & $3.5-5.1$ & 5 \\
\hline Calcium (mg/dL) & $8.6-10.2$ & 6.38 \\
\hline Phosphorus (mg/dL) & $2.45-4.5$ & 7.36 \\
\hline Uric acid $(\mathrm{mg} / \mathrm{dL})$ & $3.4-7$ & 9 \\
\hline Total bilirubin (mg/dL) & $0.1-1.2$ & 2.2 \\
\hline Direct bilirubin $(\mathrm{mg} / \mathrm{dL})$ & $0.0-0.3$ & 1.7 \\
\hline LDH (U/L) & $135-225$ & 397 \\
\hline $\operatorname{AST}(\mathrm{U} / \mathrm{L})$ & $0-40$ & 114 \\
\hline $\operatorname{ALT}(\mathrm{U} / \mathrm{L})$ & $0-41$ & 75 \\
\hline CK (U/L) & $0-190$ & 3114 \\
\hline C-reactive protein $(\mathrm{mg} / \mathrm{L})$ & $0-5$ & 417.7 \\
\hline $\operatorname{ESR}(\mathrm{mm} / \mathrm{h})$ & $0-15$ & 46 \\
\hline Procalcitonin $(\mu \mathrm{g} / \mathrm{L})$ & $<0.05$ & $>100$ \\
\hline INR (INR) & $0.8-1.2$ & 1.44 \\
\hline aPTT (sn) & $25-36$ & 43.7 \\
\hline $\mathrm{pH}$ & $7.35-7.45$ & 7.28 \\
\hline $\mathrm{PCO}_{2}(\mathrm{mmHg})$ & $35-45$ & 28.9 \\
\hline $\mathrm{HCO}_{3}(\mathrm{mmol} / \mathrm{L})$ & $22-26$ & 14.9 \\
\hline Lactate $(\mathrm{mmol} / \mathrm{L})$ & $0.7-1.8$ & 5.2 \\
\hline
\end{tabular}

LDH: lactate dehydrogenase, AST: aspartate aminotransferase, ALT: alanine aminotransferase, CK: creatine kinase, ESR: erythrocyte sedimentation rate, INR: International normalized ratio, aPTT: activated partial thromboplastin time, PCO2: partial carbon dioxide pressure, $\mathrm{HCO} 3$ : bicarbonate 
mean arterial pressure was $<65 \mathrm{mmHg}$ despite fluid resuscitation, noradrenaline infusion was commenced, and meropenem, teicoplanin, and tetracycline were administered within an hour of the patient's presentation. Due to acute renal injury, metabolic acidosis, and an elevated level of serum creatine kinase, he underwent hemodialysis. Methyl prednisolone $(1 \mathrm{mg} / \mathrm{kg})$ was administered intravenously, the lesion was cleaned with soap and water, and cold compression was applied to the lesion site. He received 3 sessions of hyperbaric oxygen as an adjunctive treatment. He also received 5 sessions of therapeutic apheresis (TPE) with a diagnosis septic shock. By day 10 of ICU admission, urine output had normalized, as had level of serum creatinine and all infection parameters; thus, noradrenalin treatment was discontinued, orthopedics performed skin debridement for the skin lesion (Figure 2), and he was transferred to the internal medicine ward on day 11 of ICU admission. On day 17 of hospital admission, a second debridement was performed due to the lesion's lack of recovery. One month after hospital admission, a skin auto-graft was performed via reconstructive and aesthetic surgery, and he was discharged from hospital on day 35 of hospital admission.

Follow-up and outcomes. It was observed that the lesion showed significant improvement in the outpatient clinic follow-up in the first month after discharge (Figure 3). Figure 4 shows timeline flowchart of the patient.

Discussion. Generally present in dark places, including lofts, basements, and beneath rocks and trees, $L$. reclusa are known to bite humans, most often

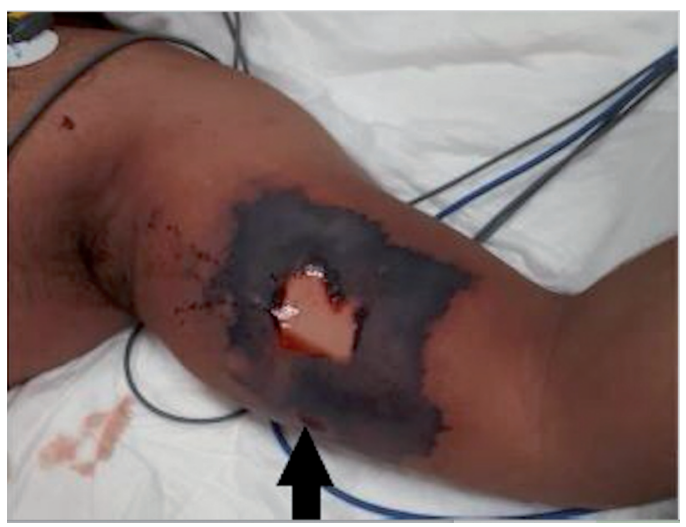

Figure 1 - The lesion image in the intensive care unit admission. The lesion $10 \times 20 \mathrm{~cm}$ with central necrosis, peripheral erythema, and edema in the middle third of the left upper arm.

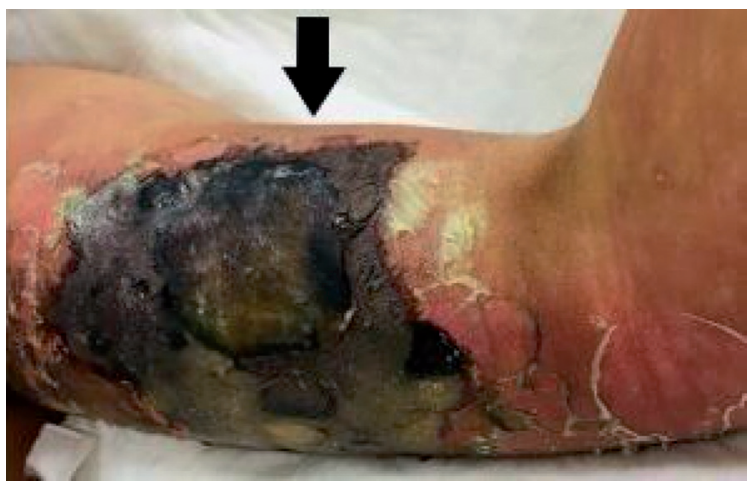

Figure 2 - The lesion image after skin debridement.

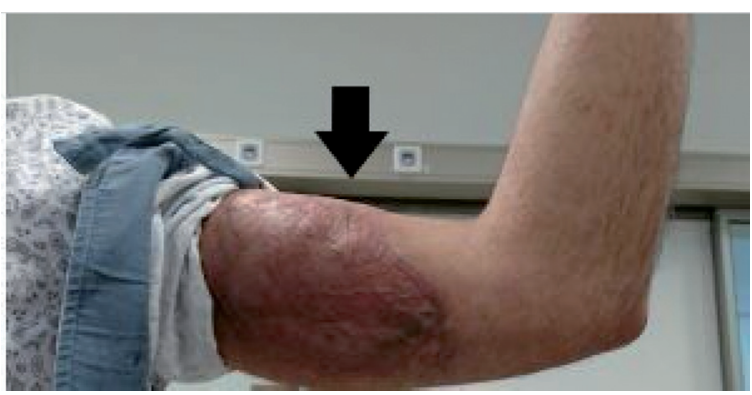

Figure 3 - The image of the lesion 35 days after skin auto-graft.

between April and October. In our case, the patient worked outdoors and presented with spider bite in June. Loxosceles reclusa venom, both hemolytic and cytotoxic, is the most potent of all spider venoms and contains several enzymes that cause clinical symptoms. ${ }^{1}$ Among them, sphingomyelinase $\mathrm{D}$ governs toxin-mediated hemolysis and complement-mediated erythrocyte destruction and can cause cutaneous conditions by way of synergistic effects. ${ }^{2}$ The venom also contains proteases that break down collagen, fibronectin, fibrinogen, and elastin-basement membranes.

Spider intoxication is termed arachnidism if it causes local tissue injury and loxoscelism if it causes systemic syndrome. ${ }^{3}$ On some occasions, only urticarial rash develops due to spider bites, and though systemic involvement is rare, it did affect our patient. Although spider bites are initially painless, pain can emerge within 2 to 8 hours. In loxoscelism in particular, fatigue, nausea, vomiting, diarrhea, fever up to $40.5^{\circ} \mathrm{C}$, chills, headache, myalgia, arthralgia, hemoglobinuria, hematuria, proteinuria, intravascular coagulation, acute renal injury, rhabdomyolysis, pyoderma gangrenosum, and pulmonary edema can develop. ${ }^{4}$ 
Relevant Past Medical History

Male, 24 years old, was previously healthy
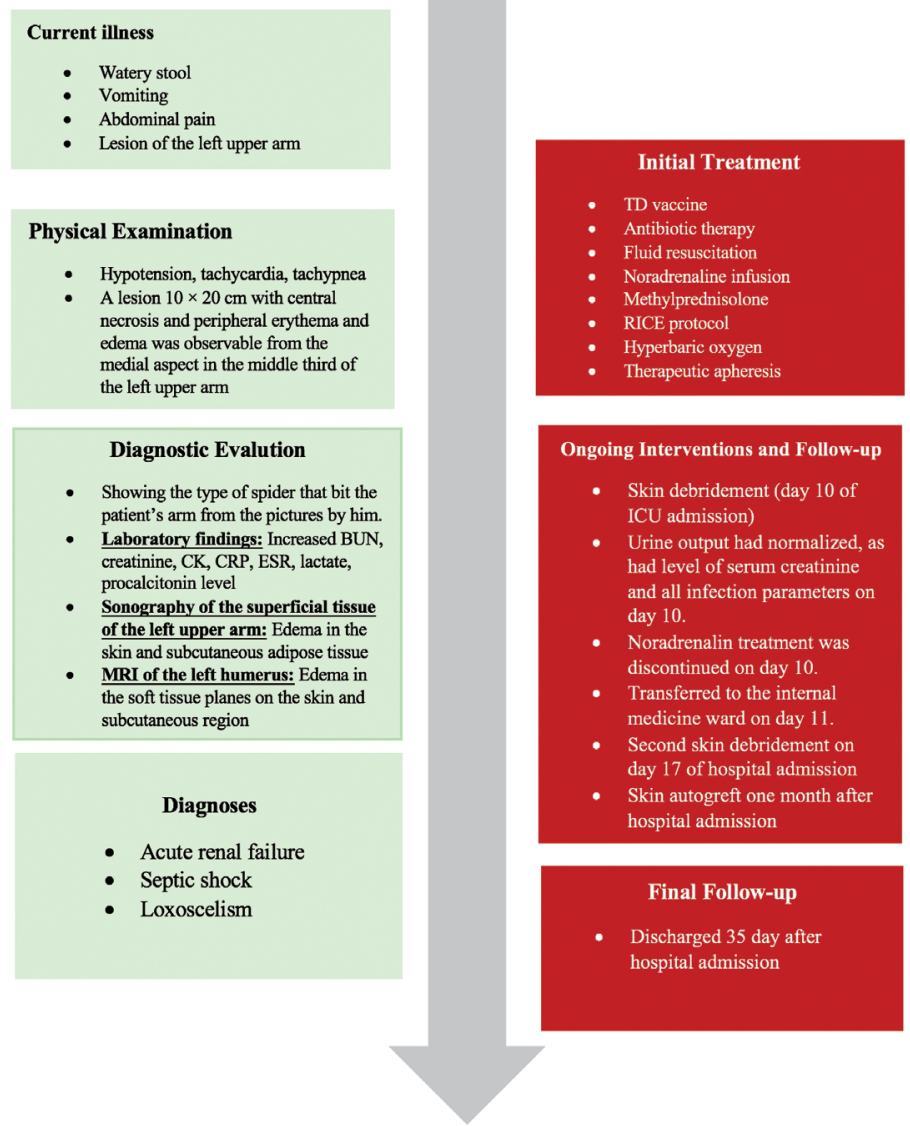

Resolution of This Episode of Care

- The patient's lesion exhibited marked recovery one month after the discharged

Figure 4 - Timeline flowchart of the patient with a diagnosis Loxoscelism. BUN: blood urea nitrogen, CK: Creatine kinase, CRP: C-reactive protein, ESR: erythrocyte sedimentation rate, MRI: magnetic resonance imaging, TD: tetanus and diphtheria, RICE: Rest, ice, compression, and elevation.

Our patient, whose presented complaints were nausea, vomiting, and diarrhea, later showed acute renal injury, thrombocytopenia, and rhabdomyolysis. During the first hours following spider bite, 2 small holes surrounded by erythema may occur, and over time, peripheral edema and erythema may develop around the wound while the center fades, which enhances vasospasm and, in turn, intensifies pain. Blisters typically develop within a few days, with centers that are solid and become star-like purplish-blue areas, after which skin exchange can occur, and the wound can heal aided by secondary intervention. Grayish or black skin lesions indicate necrotizing fasciitis. In response, computed tomography scanning or MRI is recommended for patients at high risk of necrotizing fasciitis. The LRINEC scoring system, designed to predict necrotizing fasciitis, offers scores from 0 to 13 . The scores of 6 or less indicate necrotizing fasciitis, whereas scores of 8 or greater are highly predictive of necrotizing fasciitis. ${ }^{5}$ In our patient, MRI was performed as his LRINEC score was 8.

When the spider responsible for the bite is identified, the diagnosis of loxoscelism can be ascertained. Alternatively, definitive diagnosis is possible by using a sensitive enzyme-linked immunosorbent assay (ELISA) designed to detect Loxosceles venom using a specimen obtained by swabbing the lesion. However, the use of 
ELISA has been reported in only $4.2 \%$ of cases, and no assay for humans is commercially available. Thus, the diagnosis of loxoscelism is generally made by anamnesis and clinical findings. Once the wound is cleaned using soap and water, first-line therapy involves allowing the lesion site to rest, applying a cold compress to the lesion area, and elevating the extremity, all following the RICE protocol (rest, ice, compression, and elevation). Tetanus and diphtheria vaccine and antihistamines are also recommended, along with nonsteroidal antiinflammatory drugs and opiates for pain relief and antibiotics such as dapsone in the case of cellulitis or to avoid complications of infection. Although dapsone can be used to prevent neutrophil chemotaxis and reduce skin necrosis, it is harmful in patients with glucose-6-phosphate dehydrogenase (G6PD) deficiency and can cause hypersensitivity reactions. ${ }^{6,7}$ In systemic loxoscelism, anti-venom is applied in some regions; however, evidence supporting the use of dapsone and anti-venom remains controversial. ${ }^{8}$ In any case, symptomatic therapy should be provided to patients with systemic symptoms. Steroids can also decrease hemolysis and prevent renal failure due to loxoscelism, while hyperbaric oxygen therapy can be used for anaerobic soft tissue infections, especially severe ones. ${ }^{1,8}$ Hyperbaric oxygen therapy indirectly improves the activity of polymorphonuclear leukocytes, because the toxic effect of oxygen directly affects anaerobic bacteria. ${ }^{4,8}$ Also, the activity of some antibiotics increases when exposed to oxygen. The reason hyperbaric oxygen is used in patients with loxoscelism is that this treatment inactivates this enzyme by oxidizing the sulfhydryl bonds of sphingomyelinase D. ${ }^{8}$ For those reasons, we administered hyperbaric oxygen therapy to our patient. Another possible treatment is surgical excision, which should be considered after the formation of the demarcation line. Early surgical debridement is not recommended, however, a skin graft may be needed after 4 to 6 weeks. In our patient, surgical debridement was applied after the formation of the demarcation line, and a skin graft was applied one month after hospitalization. Hemodialysis should also be applied to patients with metabolic acidosis, rhabdomyolysis, or acute renal injury, if indicated, as was the case with our patient, and antibiotics, fluid resuscitation, and vasopressor therapy, if needed, should be provided to patients with sepsis or septic shock. The TPE is an extracorporeal treatment method that eliminates abnormal cells or substances that cause disease in the blood. According to the American Society of Apheresis, TPE can additionally be used as complementary treatment in sepsis with multi-organ failure (recommendation: Category III, Evidence 2B). ${ }^{9}$ Able to remove toxins, TPE was used to prevent the harmful effects of toxins to our patient, who presented with severe clinical manifestations. Cases of loxoscelism requiring TPE are rare in the literature. ${ }^{10}$

In conclusion, because the early diagnosis of spider bites can facilitate multidisciplinary, live-saving approaches to treatment, loxoscelism should be considered in patients with skin necrosis, acute renal injury, and rhabdomyolysis.

Acknowledgment. The authors gratefully acknowledg EditMyEnglish (www.editmyenglish.com) for English language editing.

\section{References}

1. Hadanny A, Fishlev G, Bechor Y, Meir O, Efrati S. Nonhealing wounds caused by brown spider bites: application of hyperbaric oxygen therapy. Adv Skin Wound Care 2016; 29: 560-566.

2. Manzoni-de-Almeida D, Squaiella-Baptistão CC, Lopes $\mathrm{PH}$, van den Berg CW, Tambourgi DV. Loxosceles venom sphingomyelinase D activates human blood leukocytes: role of the complement system. Mol Immunol 2018; 94: 45-53.

3. Chaves-Moreira D, Senff-Ribeiro A, Wille ACM, Gremski LH, Chaim OM, Veiga SS. Highlights in the knowledge of brown spider toxins. J Venom Anim Toxins Incl Trop Dis 2017; 23: 6.

4. Davanzo F, Rebutti I, Panzavolta GF, Morina M, Fumagalli R, Scagilone F, et al. Loxoscelism: proposal of a new protocol for treatment. J Clin Case Rep 2016: 6; 1000738.

5. Bechar J, Sepehripour S, Hardwicke J, Filobbos G. Laboratory risk indicator for necrotising fasciitis (LRINEC) score for the assessment of early necrotising fasciitis: a systematic review of the literature. Ann R Coll Surg Engl 2017; 99: 341-346.

6. Belfield KD, Tichy EM. Review and drug therapy implications of glucose-6-phosphate dehydrogenase deficiency. Am J Health Syst Pharm 2018; 75: 97-104.

7. Shukkoor AA, Thangavelu S, George NE, Priya S. Dapsone hypersensitivity syndrome (dhs): a detrimental effect of dapsone? a case report. Curr Drug Saf 2019; 14: 37-39.

8. Lopes PH, Squaiella-Baptistão CC, Marques MOT, Tambourgi DV. Clinical aspects, diagnosis and management of Loxosceles spider envenomation: literature and case review. Arch Toxicol 2020; 94: 1461-1477.

9. Padmanabhan A, Connelly-Smith L, Aqui N, Balogun RA, Klingel R, Meyer E, et al. Guidelines on the use of therapeutic apheresis in clinical practice- evidence-based approach from the writing committee of the American Society for Apheresis: The eighth special issue. J Clin Apher 2019; 34: 171-354.

10. Abraham M, Tilzer L, Hoehn KS, Thornton SL. Therapeutic Plasma exchange for refractory hemolysis after brown recluse spider (loxosceles reclusa) envenomation. J Med Toxicol 2015; 11: 364-367. 